\title{
Deep Learning
}

\author{
Nikita Patil ${ }^{1}$, Krishna Kadam ${ }^{2}$, Amol Kode $^{3}$, Rohit Thakur ${ }^{4}$, Rahul Patil ${ }^{5}$ \\ Students, Computer Technology, Bharati Vidyapeeth College of Technology, Kharghar, India ${ }^{1,2,3,4}$ \\ Professor, Computer Technology, Bharati Vidyapeeth College of Technology, Kharghar, India ${ }^{5}$
}

\begin{abstract}
Deep learning is used to identify the objects with the help of neural network. It is used to identify various objects in the world. In this, we have explained the process of identifying the objects with the help of various layers. Machine uses different layers to identify the object with the help of hidden layers which is used to train the machine depending upon the database provided to it. The more the database provided to it the more the accurate result the machine will provide.
\end{abstract}

Keywords: Deep Learning, machine learning, neural network

\section{INTRODUCTION}

Connectionist architectures have existed for more than 70 years but new architectures and Graphical Processing Units (GPUs) brought them to the forefront of artificial intelligence. The last two decades for deep learning architecture was greatly expanded the number and type of problems neural networks can address. Deep learning architecture are divided into several architecture such as Recurrent Neural Networks (RNNs), long short-term memory (LSTM) / Gated Recurrent Unit (GRU), Convolution Neural Networks (CNNs), Deep Belief Networks (DBN), and Deep Stacking Networks (DSNs) and then explores open source software options for deep learning. Deep learning is not a single approach but a combination of different algorithm. It is a combination of network that contains different nodes in neural network where each node in a network is called neurons.

\section{LITERATURE REVIEW}

This paper deals with the field of computer vision, focusing on the application of deep learning in object detection task ${ }^{[1]}$. On the one hand, it is a simple summary of the datasets and deep learning algorithms commonly used in computer vision. On the other hand, a new dataset is built according to commonly used datasets, and choose one of the network called faster r-cnn to work on this new dataset.

Over the years during neural networks it has shown state-of-art performance on many problems. However, their performance on recognition system is still not widely accepted ${ }^{[2]}$ in the machine learning community because these networks are unable to handle selectivity-invariance dilemma and also suffer from the problem of vanishing gradients. Some of these issues are addressed by deep learning.

Deep learning algorithms are a sub branch of the machine learning algorithms, which are used for discovering multiple levels of distributed representations. Recently, numerous deep learning algorithms have been proposed to solve traditional artificial intelligence problems ${ }^{[3]}$. This work aims to review the state-of-the-art in deep learning algorithms in computer vision by highlighting the contributions and challenges from recent research papers.

Deep learning allows automatically learning multiple levels of representations of the underlying distribution of the data to be modelled ${ }^{[4]}$. In this work, a specific implementation known as stacked demising auto encoders is explored.

\section{METHODOLOGY}

Machine learning is typically divided into supervised learning, where the computer learns from the example given as labeled data, and unsupervised learning, where the computer groups similar data and pinpoints inconsistency. Deep learning is a sub branch of machine learning, whose capabilities differ in several key respects from traditional deep machine learning, allowing computers to solve a host of difficult problems that couldn't otherwise be tackled.

An example of a simple, deep machine-learning task might be predicting how ice-cream sales will vary based on outdoor temperature. Making predictions using only a couple of data features in this way is comparatively straightforward, and can be carried out using a deep machine-learning technique called linear regression with gradient 
descent. The issue is that wrap of problems in the real world aren't a good fit for such simple models. An example of one of these compound real-world problems is recognizing handwritten numbers.

To solve this problem, the computer needs to be able to manage with huge variety in how the data can be presented. Every digit between 0 and 9 can be written in different ways: the size and exact shape of each handwritten digit can be very different depending on who's writing and in what circumstance. Coping with the variability of these features, and the even bigger disorder of interactions between them, is where deep learning and deep neural networks become useful. Neural networks are mathematical models whose structure is inspired by that of the brain. Each neuron within a neural network is a mathematical function that takes in data via an input, converts that data into a more manageable form, and then gives it out via an output. You can think of neurons in a neural network as being arranged in each layer, as shown below.

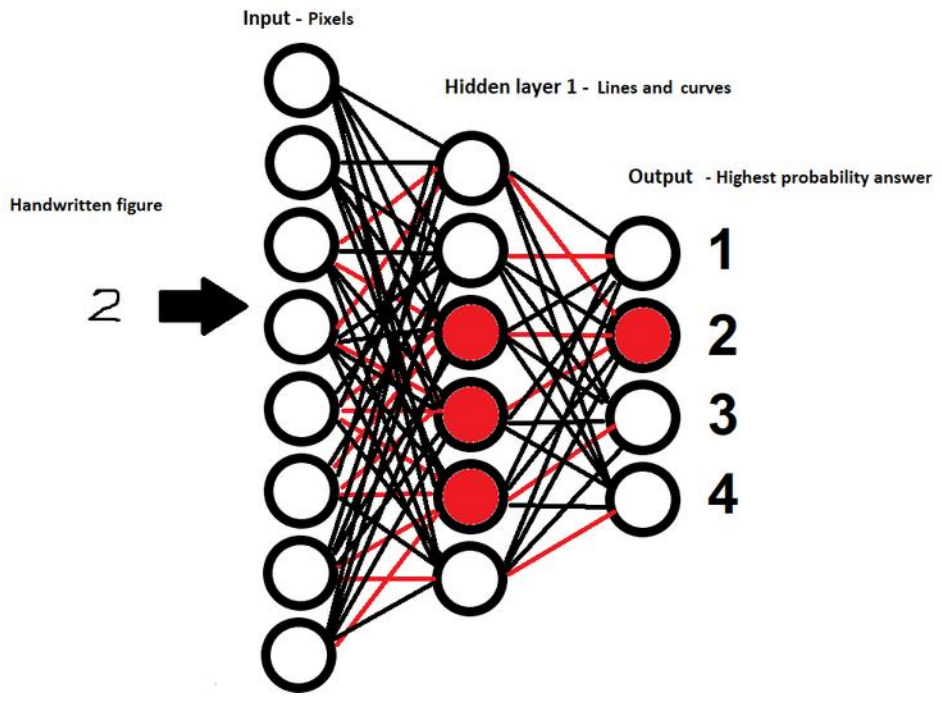

All neural networks have an input layer, where the initial data is given in, and an output layer, that generates the final prediction. But in a deep neural network, there will be multiple "hidden layers" of neurons between these input and output layers, each giving data into each other. Hence the term "deep" in "deep learning" and "deep neural networks", it is a reference to the large number of invisible layers -- typically greater than three -- at the heart of these neural networks. This simplified diagram above hopefully helps to provide an idea of how a simple neural network is arranged. In this example, the network has been trained to recognize handwritten figures, such as the number 2 shown here, with the input layer being given values representing the pixels that creates an image of a handwritten digit, and the output layer predicting which handwritten number was shown in the image.

In the diagram shown above, each circle represents a neuron in the network, with the neurons organized into vertical layers. As you can see, each neuron is linked to every neuron in the following layer, representing the fact that each neuron outputs a value into every neuron in the following layer. The color of the links in the diagram also differs. The different colors, black and red, represent the importance of the links between neurons. The red links are those of greater importance, meaning they will increase the value as it passes between the layers. In turn, this expansion of the value can help activate the neuron that the value is being given into.

A neuron can be said to have been activated when the sum of the values being input into this neuron passes a set entrance. In the diagram, the activated neurons are colored red. In "Hidden layer 1" shown in the diagram, an activated neuron maybe mean the image of the handwritten figure contains a certain combination of pixels that may recognize the horizontal line at the top of a handwritten number i.e. 7. In this way, "Hidden layer 1" could detect many of the lines and curves that will eventually combine together into the full handwritten figure.

An actual neural network would likely have both more invisible layers and more neurons in each layer. For instance, a "Hidden layer 2" could be given the small lines and curves identified by "Hidden layer 1", and detect how these combine to form identifiable shapes that create the digits, such as the entire bottom loop of a six. By giving data forward between layers in this way, each following hidden layer handles increasingly higher-level features.

By building up a picture of which of these features, modern neural networks can check -- with a very high level of accuracy -- the number that corresponds to a handwritten digit. Similarly, different types of deep neural networks can be trained to recognize faces in an image or to reproduce written speech from audio. The process of building this increasingly compound hierarchy of features of the handwritten number out of nothing but pixels is learned by the 
network. The learning process is made possible by how the network is able to differ the importance of the links between the neurons in each layer. Each link has a value called a weight, which will modify the value out by a neuron as it passes from one layer to the next. By altering the value of these weights, and an associated value called bias, it is possible to highlight the importance of links between neurons in the network.
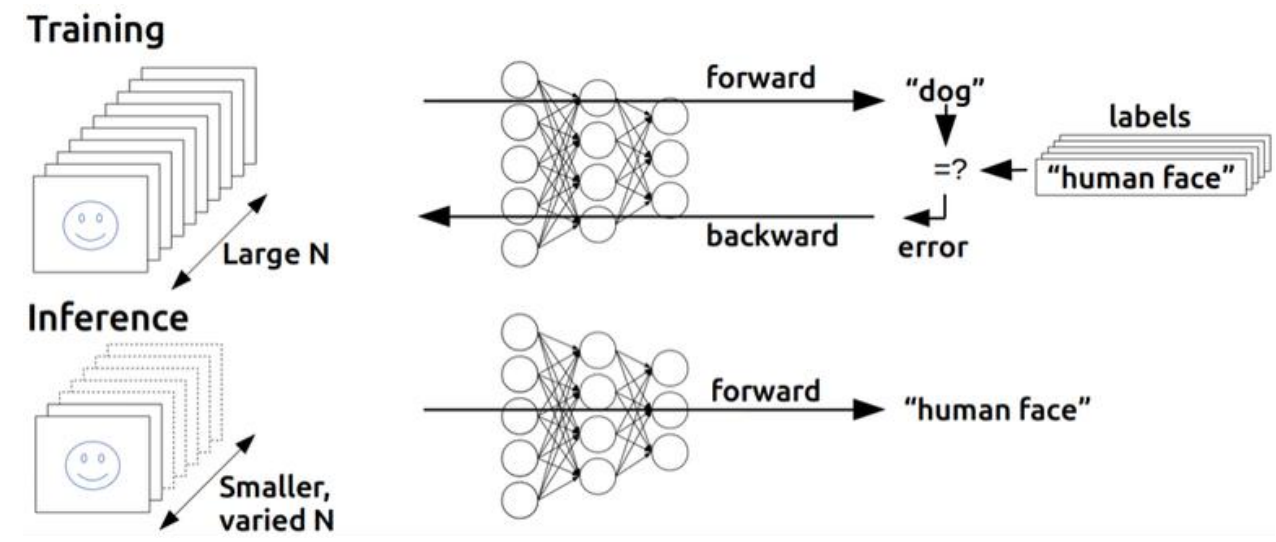

The model learns which links are important in making successful predictions during training. At each step during training, the network will use a mathematical function to determine how correct its latest prediction was compared to what was expected. This function generates a series of error values, which in turn can be used to calculate how the model should update the value of the weights attached to each link, with the aim of improving the accuracy of the network's predictions. The size to which these values should be changed is calculated by a development function, such as inclined descent, and those changes are pushed back all over the network at the end of each training cycle called propagation.

Over the course of many, many training cycles, and with the help of irregular manual parameter tuning, the network will continue to generate better and better predictions until it hits close to accurate result. At this point, for example, when handwritten digits could be identified with more than 95 percent accuracy, the deep-learning model can be said to have been trained successfully. Essentially deep learning allows machine learning to implement a whole host of new compound problems such as image, language and speech recognition by allowing machines to learn how features in the data combine into increasingly higher level, abstract forms. For example in facial recognition, how pixels in an image create lines and shapes, and depending on it identify the image of the person.

\section{IV.CONCLUSION}

In this we have studied and gathered the information about deep learning and how it is going to change the world in the future. Deep learning is going to be used to detect many more things like face recognition, handwriting detection, detecting the food and everything else. Deep learning is deeply linked with the neural network which helps to identify the objects easily.

\section{REFERENCES}

[1]. "Overview of deep learning" Xuedan Du, Yinghao Cai and Shuo Wang https://ieeexplore.ieee.org/document/7804882/

[2]. "Application of deep learning in object detection" Xinyi Zhou, Wei Gong, WenLong Fu and Fengtong Du https://ieeexplore.ieee.org/ document/7960069/

[3]. "A review on advances in deep learning" Soniya, Sandeep Paul and Lotika Singh https://ieeexplore.ieee.org/document/7495514/

[4]. "Image recognition method based on deep learning" Xin Jia https://ieeexplore.ieee.org/document/7979332/

[5]. "An introduction to deep learning" Francis Quintal Lauzon https://ieeexplore.ieee.org/document/6310529/

[6]. "Efficient Processing of Deep Neural Networks: A Tutorial and Survey" Vivienne Sze, Yu-Hsin Chen, Tien-Ju Yang and Joel S. Emer https: //ieeexplore .ieee.org/document/8114708/

[7]. "Artificial intelligence, machine learning and deep learning" Pariwat Ongsulee https://ieeexplore.ieee.org/document/8259629/

[8]. "Deep learning" Yann LeCun, Yoshua Bengio and Geoffrey Hinton https://www.nature.com/articles/nature14539

[9]. Li Deng and Dong Yu (2014), "Deep Learning: Methods and Applications", Foundations and Trends® in Signal Processing: Vol. 7: No. 3-4, pp 197-387. http://dx.doi.org/10.1561/2000000039

[10]. "Deep Learning" Kwang Gi Kim https://www.synapse.koreamed.org/Synapse/Data/PDFData/1088HIR/hir-22-351.pdf

[11]. "Deep Learning-Based Classification of Hyperspectral Data" Yushi Chen, Zhouhan Lin, Xing Zhao, Gang Wang and Yanfeng Gu https:// ieexplore.ieee.org/abstract/document/6844831/

[12]. "Traffic Flow Prediction With Big Data: A Deep Learning Approach" Yisheng Lv, Yanjie Duan, Wenwen Kang, Zhengxi Li, Fei-Yue Wang https://ieeexplore.ieee.org/document/6894591/ 